\title{
Influência da temperatura de pirólise nas emissões gasosas, rendimentos e densidade do carvão vegetal
}

\author{
Júlia Melo Franco Neves Costa ${ }^{1}$, Angélica de Cássia Oliveira Carneiro ${ }^{2}$, Ana Márcia Macedo Ladeira \\ Carvalho $^{2}$, Laercio Antônio Gonçalves Jacovine ${ }^{2}$, Benedito Rocha Vital ${ }^{2}$, Solange de Oliveira Araujo ${ }^{3}$, \\ Wagner Davel Canal ${ }^{4}$
}

\author{
${ }^{1}$ SUPRAM Jequitinhonha, Minas Gerais, Brasil. \\ ${ }^{2}$ Universidade Federal de Viçosa, Viçosa, Minas Gerais, Brasil. \\ ${ }^{3}$ Universidade Técnica de Lisboa, Lisboa, Portugal. \\ ${ }^{4}$ Secretaria de Educação do Estado do Espírito Santo, Espírito Santo, Brasil.
}

\begin{abstract}
RESUMO A maximização da temperatura de carbonização não deve ser vista unicamente como a solução imediata para se obter um produto com bons rendimentos, pois tal ação tem a capacidade de impactar sobremaneira a qualidade do ambiente. Avaliou-se o efeito de incrementos em temperatura nas emissões de gases, rendimentos dos produtos da carbonização e densidade do carvão vegetal. Utilizou-se madeira de Eucalyptus sp., com aproximadamente 8 anos de idade, para carbonização em mufla nas temperaturas de 400,550 e $700{ }^{\circ} \mathrm{C}$, coletados e analisados os gases $\mathrm{CO}_{2}, \mathrm{CO}, \mathrm{CH}_{4}$ e $\mathrm{H}_{2}$. Determinou-se o rendimento gravimétrico dos produtos e a densidade aparente do carvão. $\mathrm{O}$ aumento da temperatura até $700{ }^{\circ} \mathrm{C}$ proporcionou a diminuição do rendimento em carvão, aumentos em gases condensáveis e não condensáveis. Houveram picos de emissão dos gases $\mathrm{CO}_{2}$ e CO partir de 200 até temperaturas próximas de $450{ }^{\circ} \mathrm{C}$; houve liberação dos gases $\mathrm{H}_{2}$ e $\mathrm{CH}_{4}$, desde 300 até de 500 e $550^{\circ} \mathrm{C}$, respectivamente. $\mathrm{O}$ aumento da temperatura proporcionou aumentos nas emissões de praticamente todos os Gases de Efeito Estufa (GEE). Conclui-se que a geração dos gases $\mathrm{CO}_{2}$ e $\mathrm{CO}$ tem tendência de estabilização e a geração dos gases $\mathrm{H}_{2} \mathrm{e}$ $\mathrm{CH}_{4}$ apresenta crescimento progressivo com o aumento da temperatura; aumentos na temperatura promovem aumentos nos rendimentos gravimétricos em gases condensáveis e gases não condensáveis e diminuição no rendimento gravimétrico em carvão vegetal; e os incrementos em temperatura não influenciaram na obtenção de carvão vegetal com diferentes densidades. Palavras-chave: aumento da temperatura; rendimentos gravimétricos em gases; qualidade do ambiente.
\end{abstract}

\section{Influence of pyrolysis temperature in gas emissions, yields and coal density}

\begin{abstract}
Maximizing the carbonization temperature should not only be seen as the immediate solution to produce a product with good yields, as such action has the ability to greatly impact the quality of the environment. The effect of temperature increases on gas emissions, carbonization product yields and charcoal density was evaluated. Eucalyptus sp. wood, with approximately 8 years old, was used on muffle carbonization at temperatures of 400,550 and $700{ }^{\circ} \mathrm{C}$, and $\mathrm{CO}_{2}, \mathrm{CO} \mathrm{CH}_{4}$ and $\mathrm{H}_{2}$ gases were collected and analyzed. The gravimetric yield of the products and the bulk density of the coal were determined. Increasing the temperature to $700^{\circ} \mathrm{C}$ led to decreased coal efficiency, increases in condensable and non-condensable gases. There were emission peaks of $\mathrm{CO}_{2}$ and $\mathrm{CO}$ from 200 to temperatures around $450{ }^{\circ} \mathrm{C} ; \mathrm{H}_{2}$ and $\mathrm{CH}_{4}$ gases were released, ranging from 300 to 500 and $550^{\circ} \mathrm{C}$, respectively. Rising temperatures have led to increases in emissions from virtually all Greenhouse Gases (GHG). It is concluded that the generation of $\mathrm{CO}_{2}$ and $\mathrm{CO}$ gases tends to stabilize and the generation of $\mathrm{H}_{2}$ and $\mathrm{CH}_{4}$ gases presents progressive growth with increasing temperature; increases in temperature promote increases in gravimetric yields in condensable gases and non-condensable gases and decrease in gravimetric yield in charcoal; and the temperature increases did not influence the charcoal production with different densities.
\end{abstract}

Keywords: temperature increase; gravimetric gas yields; quality of the environment. 


\section{Introdução}

O panorama atual da produção de carvão vegetal no Brasil baseia-se, principalmente, na utilização de fornos rudimentares de alvenaria, o que impacta em uma produção de carvão vegetal com baixos rendimentos, pouca resistência física e mecânica, além da não recuperação dos gases de efeito estufa (GEE) liberados para o ambiente durante a carbonização. Ao se considerar as inúmeras tecnologias empregadas para a produção do carvão vegetal, a temperatura final pode influenciar na dinâmica da carbonização da madeira, de modo que a densidade do carvão, as emissões gasosas e o rendimento dos produtos do processo são influenciados pelas características de resistividade térmica da madeira. Desse modo, o controle da temperatura final de carbonização da madeira é considerado fator importante para a formação de carvão vegetal em quantidade e qualidade demandadas pela siderurgia. Portanto, observa-se que de acordo com incrementos ou não da temperatura de carbonização a produção de carvão vegetal pode ser considerada fator preponderante ao equilíbrio do meio ambiente no que se refere às emissões de GEE.

No que se refere às tecnologias empregadas para a obtenção de carvão vegetal, Carneiro et al. (2013) consideram que temperaturas próximas de $400^{\circ} \mathrm{C}$ garantem a purificação da madeira com a volatilização de $89 \%$ de oxigênio e formação de um produto energético com aproximadamente 57\% de carbono. Couto et al. (2015) completam ainda que a elevação da temperatura de carbonização garante concentração de carbono durante a pirólise da madeira, gerando assim valores elevados de densidade do carvão vegetal. Desse modo, apesar de se obter uma biomassa altamente energética através do processo de carbonização e com elevados valores de densidade de acordo com incrementos em temperatura, deve-se considerar os efeitos nocivos da carbonização nas emissões de gases de efeito estufa, pois sucessivos incrementos em temperatura também influenciam na intensificação das emissões gasosas.

Diante da exposição da relação que envolve as variáveis temperatura e emissão, Duboc et al. (2007) afirmaram que são lançados aproximadamente de 65 a 70\% de material lenhoso na forma gasosa para a atmosfera no decorrer da carbonização. Klose et al. (2000) citam ainda que a porção gasosa não condensável, liberada na pirólise da madeira, é uma mistura complexa e variável, constituída prioritariamente, por dióxido de carbono $\left(\mathrm{CO}_{2}\right)$, monóxido de carbono (CO), metano $\left(\mathrm{CH}_{4}\right)$, hidrogênio $\left(\mathrm{H}_{2}\right)$ e hidrocarbonetos leves. Em virtude da purificação da madeira sob a elevação da temperatura, Becidan et al. (2007) consideram que temperaturas de carbonização superiores às praticadas na produção de carvão vegetal para a siderurgia $\left(400{ }^{\circ} \mathrm{C}\right)$ tornam o processo ainda mais impactante ao meio ambiente, devido a formação de maiores teores de gases de efeito estufa e diminuição dos rendimentos em carvão vegetal. Além disso, deve-se considerar que, para Santos et al. (2012), com os conhecimentos químicos relacionados a resistividade térmica dos componentes da madeira é possível interpretar como é o seu comportamento termodegradativo durante os incrementos em temperatura.

Assim, a maximização da temperatura da carbonização não deve ser vista unicamente como a solução imediata para se obter um produto com bons rendimentos, pois tal ação tem a capacidade de impactar sobremaneira a qualidade do ambiente. Deve-se buscar, portanto, um equilíbrio para a variável temperatura de modo a se obter um produto com qualidade para atender as exigências do setor siderúrgico, com menor perda possível em produtividade e proporcionar condições salubres ao meio ambiente. Diante do exposto, o objetivo deste estudo foi avaliar a influência da temperatura 
final durante a carbonização da madeira de Eucalyptus sp. na composição dos gases não condensáveis, no rendimento dos produtos da carbonização e na densidade do carvão vegetal.

\section{Materiais e Métodos}

O presente estudo foi conduzido no Laboratório de Painéis e Energia da Madeira (LAPEM) do Departamento de Engenharia Florestal da Universidade Federal de Viçosa, situado no município de Viçosa (MG), Brasil. Para a realização deste trabalho, utilizou-se madeira de Eucalyptus sp. com aproximadamente oito anos de idade.

\section{Carbonização da madeira, rendimentos}

\section{densidade do carvão vegetal}

Foram selecionadas aleatoriamente 2 (duas) toras de um montante de 20 (vinte) estéreos de madeira. De cada tora foram retirados 5 (cinco) toretes com aproximadamente 5 (cinco) centímetros de espessura, seccionados ao longo de toda a sua altura. Posteriormente, as amostras de madeira foram transformadas em cavacos, misturadas e secas em estufa $\left(105 \pm 3^{\circ} \mathrm{C}\right)$ para se realizarem carbonizações em forno elétrico tipo mufla. Para as carbonizações, $250 \mathrm{~g}$ de madeira na forma de cavacos foram submetidas aos seguintes incrementos em temperatura descrito na Tabela 1.

Para cada temperatura final, realizaram-se incrementos de $50^{\circ} \mathrm{C}$ a cada 30 minutos e posterior estabilização de 30 minutos antes da finalização do processo para que houvesse a certificação de que a carbonização atingisse as temperaturas finais sugeridas, conforme também utilizado por Protásio et al. (2015).

Após as carbonizações foram determinados os rendimentos gravimétricos em carvão vegetal (equação 1), em gases condensáveis (equação 2) e em gases não condensáveis (equação 3), em relação à massa seca de madeira. A densidade do carvão, a $0 \%$ de umidade, foi determinada pelo método hidrostático, por meio da imersão em mercúrio, conforme descrito por Vital (1984). Os valores médios dos rendimentos dos produtos da pirólise são apresentados nas Equações 1, 2 e 3.

$$
\begin{aligned}
R G C & =\frac{M C V}{M m a d s} & & \text { (Equação 1) } \\
R G G C & =\frac{M G C}{M m a d s} & & \text { (Equação 2) } \\
R G G N C & =R G C-R G G C & & \text { (Equação 3) }
\end{aligned}
$$

Em que: $\mathrm{RGC}=$ rendimento gravimétrico em carvão vegetal; RGGNC = rendimento gravimétrico em gases condensáveis; $\mathrm{MCV}$ = massa de carvão vegetal, em g; MGC = massa de gases condensáveis ou licor pirolenhoso, em g; Mmads $=$ massa de madeira seca, em g.

\section{Sistema de coleta de gases não condensáveis}

Observa-se na Figura 1 o sistema de coleta de gases não condensáveis (GNC):

Conforme as considerações realizadas por Canal et al. (2016), o sistema de coleta de gases não condensáveis era composto por uma bomba com vazão de coleta ajustada para $1,667 \times 10^{-5} \mathrm{~m}^{3} / \mathrm{s}$, que tinha a função de succionar os gases não condensáveis da carbonização da madeira até o sistema de condicionamento de gases Gasboard 9030 Wuhan CUBIC Optoeletronics Co. LTDA

Tabela 1: Informações gerais sobre os diferentes tratamentos empregados.

Table 1. General information about the different treatments employed.

\begin{tabular}{cccc}
\hline Tratamento & Temperatura inicial $\left({ }^{\circ} \mathbf{C}\right)$ & Temperatura final $\left({ }^{\circ} \mathbf{C}\right)$ & Tempo de carbonização $(\mathbf{h})$ \\
\hline $\mathbf{1}$ & 100 & 400 & 3,5 \\
$\mathbf{2}$ & 100 & 550 & 5,0 \\
$\mathbf{3}$ & 100 & 700 & 6,5 \\
\hline
\end{tabular}




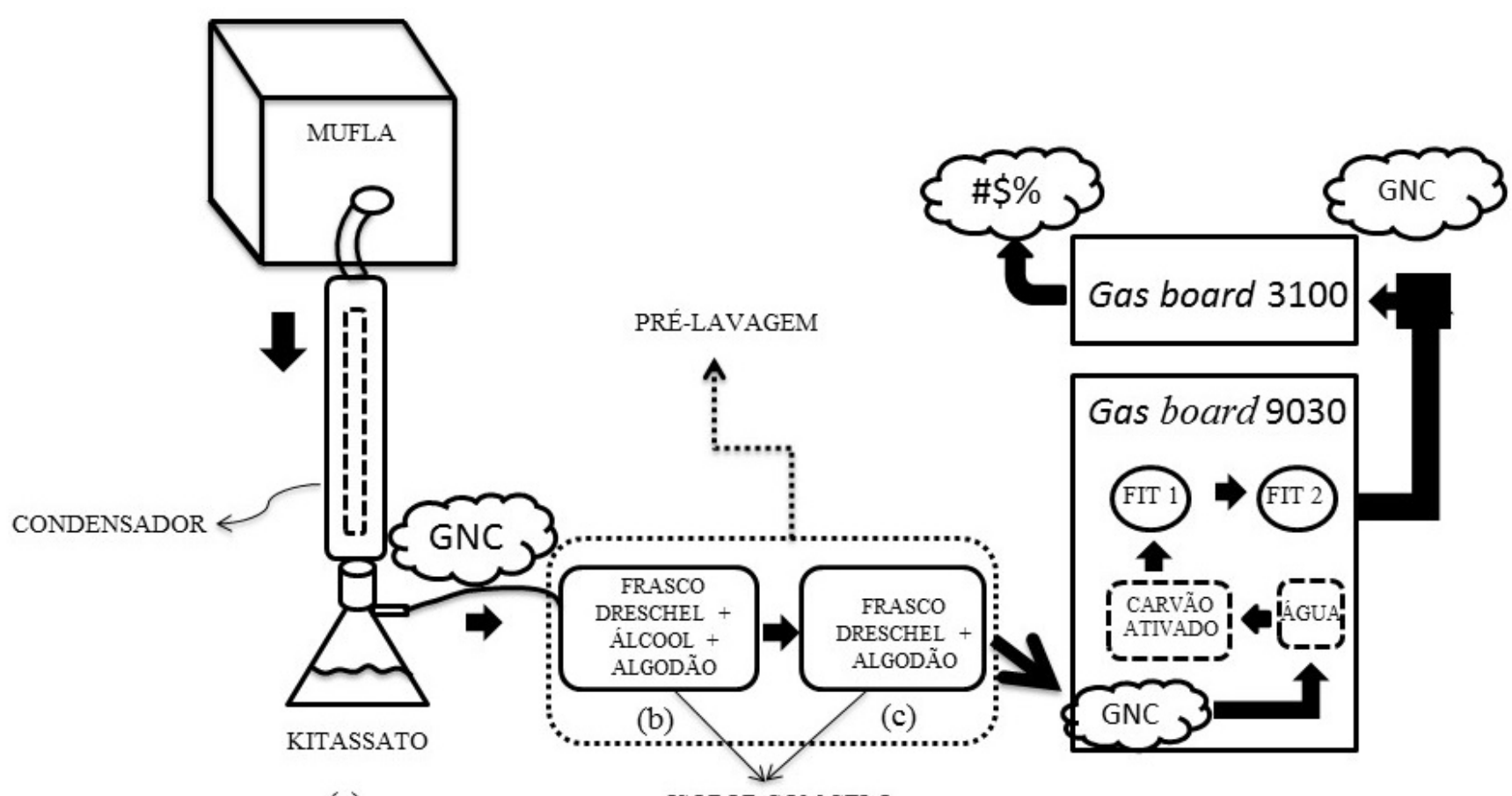

(a)

ISOPOR COM GELO

Figura 1. Caracterização do sistema de tratamento e análise dos gases não condensáveis (GNC).

Figure 1. Characterization of the processing system and analysis of non-condensable gases (NCG).

Fonte: Canal et al. (2016)

Em que: FIT 1 = filtro de precisão que retém impurezas maiores que $3 \mu \mathrm{m}$; FIT 2 = filtro de precisão que retém impurezas maiores que $1 \mu \mathrm{m}$.

composto por uma bomba com vazão de coleta ajustada para $1,667 \times 10^{-5} \mathrm{~m}^{3} / \mathrm{s}$, que tinha a função de succionar os gases não condensáveis da carbonização da madeira até o sistema de condicionamento de gases Gasboard 9030 Wuhan CUBIC Optoeletronics Co. LTDA. Essa sucção foi realizada a cada incremento de $50{ }^{\circ} \mathrm{C}$, correspondendo a intervalos de 30 minutos, até as temperaturas finais de 400,550 e $700{ }^{\circ} \mathrm{C}$. Antes da injeção no equipamento, os gases passaram por um processo de pré-lavagem, no qual foram encaminhados a um frasco coletor de gases condensáveis (Figura 1a), e mais outros dois frascos lavadores de gás do tipo Dreschel, o primeiro contendo álcool absoluto e algodão (Figura 1b) e o segundo contendo somente algodão (Figura 1c), ambos envolvidos com gelo e armazenados em isopor. A lavagem foi necessária para evitar que possíveis gotículas de alcatrão que porventura não tivessem condensado no kitassato viessem a fazê-lo na tubulação do Gasboard, saturando o aparelho.
Após esse caminho de purificação dos gases, os mesmos seguiram para o interior do aparelho Gasboard 9030 e conduzidos por outra lavagem em água, resfriamento a $4{ }^{\circ} \mathrm{C}$ num desumidificador (chiller), e posteriormente por um cilindro contendo carvão ativado e algodão. Em seguida, procedeu-se à última limpeza dos gases por meio de filtros denominados FIT1 e FIT2, descritos na legenda da Figura 1.

Concomitante ao sistema de purificação externa e interna ao aparelho de sucção, os gases seguiram até o sistema online de análise de gás, denominado Gasboard 3100 Wuhan CUBIC Optoeletronics Co (CANAL et al., 2016). Este aparelho forneceu leituras da composição percentual base volume de $\mathrm{CH}_{4}, \mathrm{CO}_{2}, \mathrm{CO}, \mathrm{H}_{2}, \mathrm{O}_{2}$, correspondendo a um total de 7, 10 e 13 registros para as temperaturas de 400, 550 e 700 ${ }^{\circ} \mathrm{C}$, respectivamente.

Com base nos dados obtidos de concentração de cada gás e o de massa dos gases não condensáveis, foi possível 
mensurar a massa total (em g) de cada componente do gás não condensável emitida nas três temperaturas finais, obter a massa total do gás não condensável liberado durante a carbonização (em g) e transformá-los em fator de emissão utilizando o balanço de massa também descrito por Canal et al. (2016). Por fim, essas emissões gasosas obtidas foram utilizadas para a confecção de curvas percentuais de liberação de cada componente gasoso mediante a evolução da temperatura.

\section{Análise dos dados}

$\mathrm{O}$ experimento foi instalado segundo um delineamento inteiramente casualizado, com três tratamentos $(400,550$ e $700{ }^{\circ} \mathrm{C}$ ) e três repetições (carbonizações), totalizando 9 unidades amostrais. Os dados foram submetidos aos testes de Lilliefors, para testar a normalidade, e Cochran, para testar a homogeneidade das variâncias. Em seguida os resultados foram submetidos à análise de variância (ANOVA), para verificação das diferenças existentes entre as temperaturas avaliadas. Quando estabelecidas diferenças significativas entre eles, aplicou-se o teste Tukey em nível de 95\% de significância, para identificar possível influência da temperatura na emissão em massa dos gases não condensáveis e na densidade do carvão.

\section{Resultados e Discussão}

\section{Quantificação e qualificação dos gases não}

\section{condensáveis}

Através das interpretações dadas no item "Sistema de coleta de gases não condensáveis", do Materiais e Métodos, considera-se que os valores médios de emissões de cada gás nas diferentes temperaturas finais se referem principalmente até a temperatura de $400{ }^{\circ} \mathrm{C}$. Isso se explica pois os valores médios de vazão de cada gás não condensável nas diferentes temperaturas finais é maior em $400{ }^{\circ} \mathrm{C}(63$ observações entre 100 e $400^{\circ} \mathrm{C}$ ), em comparação com as temperaturas finais de 550 (18 observações entre 450 e $\left.550{ }^{\circ} \mathrm{C}\right)$ e $700{ }^{\circ} \mathrm{C}(9$ observações entre 100 e $700^{\circ} \mathrm{C}$ ).

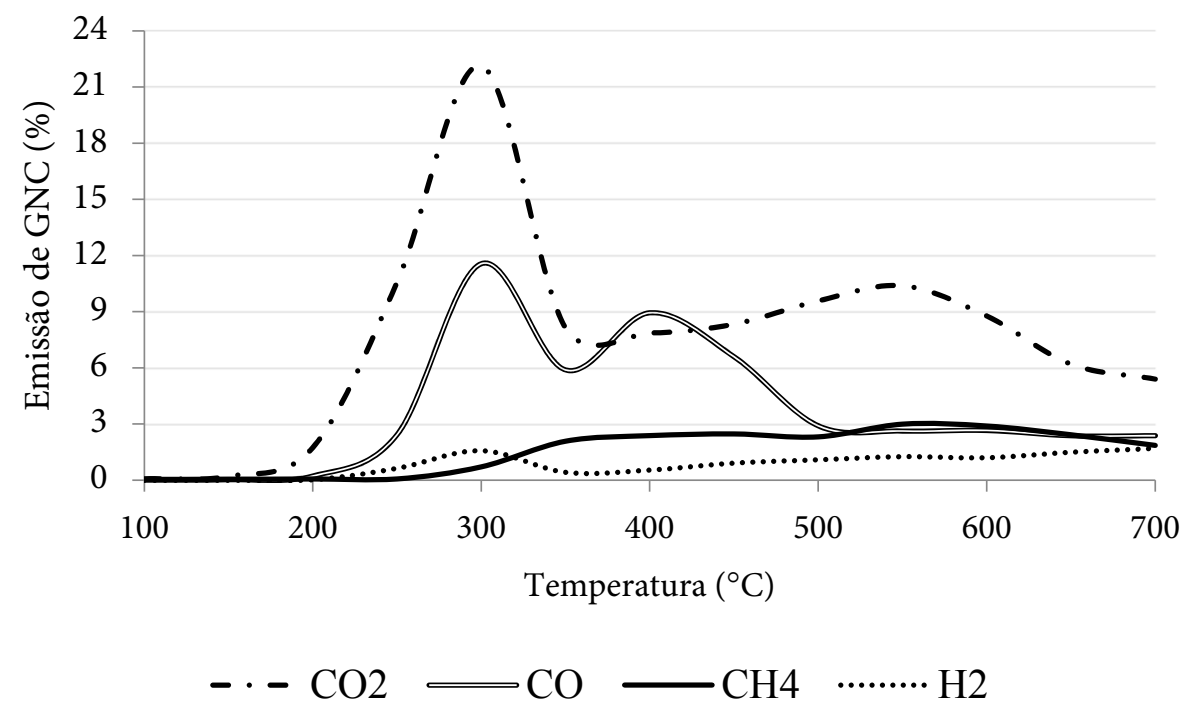

Figura 2. Composição percentual média dos gases não condensáveis da carbonização da madeira de Eucalyptus sp. Figure 2. Average percentage composition of non-condensable gases from the carbonization of Eucalyptus sp. Em que: $\mathrm{GNC}=$ Gás não condensável; $\mathrm{CO} 2=$ Dióxido de carbono; $\mathrm{CO}=$ Monóxido de carbono; $\mathrm{CH} 4=\mathrm{Metano} ; \mathrm{H} 2=$ Hidrogênio. 
Durante a fase inicial de aquecimento da madeira, que corresponde às temperaturas entre 100 e $200{ }^{\circ} \mathrm{C}$, não foram observados picos consideráveis da emissão de gases oriundos da carbonização. Para Carneiro et al. (2013) e Canal et al. (2016) esse início do processo de carbonização se deve à secagem da madeira, que ocorre até $200^{\circ} \mathrm{C}$, em que só há a liberação de vapor d'água. No entanto, como o material do presente estudo estava completamente seco ( $0 \%$ de umidade em base seca), nenhuma liberação gasosa foi evidenciada. De acordo com Raad et al. (2006), a não ocorrência da emissão de gases na faixa de temperatura citada deve-se à estabilidade térmica dos componentes químicos da madeira (celulose, hemiceluloses e lignina) por períodos curtos de exposição à temperatura.

Posteriormente, observou-se picos de emissão dos gases $\mathrm{CO}_{2}$ e $\mathrm{CO}$ a partir dos $200{ }^{\circ} \mathrm{C}$, atingindo concentrações próximas a $22 \%$ e $12 \%$, respectivamente, aos $300{ }^{\circ} \mathrm{C}$, sendo que Yang et al. (2007), Shen et al. (2010), Santos et al. (2012) e Pereira et al. (2013) atribuem esta temperatura à decomposição térmica das hemiceluloses e celulose.

Já em relação aos gases $\mathrm{H}_{2} \mathrm{e} \mathrm{CH}_{4}$, nota-se que eles têm uma menor contribuição percentual na composição dos gases não condensáveis da carbonização. $\mathrm{O} \mathrm{H}_{2}$ começa a ter uma liberação mais significativa próxima dos $500^{\circ} \mathrm{C}$, com alcance de $1,7 \%$ aos $700{ }^{\circ} \mathrm{C}$, porém, apresentou um pico de concentração isolado em temperatura inferior, com valor de $1,5 \%$ aos $300{ }^{\circ} \mathrm{C}$. Já para o $\mathrm{CH}_{4}$, notou-se uma evolução contínua a partir dos $300{ }^{\circ} \mathrm{C}$, chegou aos $3 \%$ na temperatura de $550^{\circ} \mathrm{C}$ e estabilizou-se até o fim da carbonização.

Em virtude dessas liberações gasosas diferenciadas ao longo dos incrementos em temperatura, Shen et al. (2010) consideram que a degradação completa das hemiceluloses e celulose ocorre até temperaturas limítrofes de $400{ }^{\circ} \mathrm{C}$ (com maior produção de $\mathrm{CO}_{\text {e }} \mathrm{CO}_{2}$ ) e a decomposição térmica da lignina acontece lentamente desde temperaturas próximas a 400 até $900{ }^{\circ} \mathrm{C}$ (liberação contínua de $\mathrm{CH}_{4}$ e $\mathrm{H}_{2}$ ).

Segundo Pereira et al. (2013), a temperatura em torno de 400 e $450{ }^{\circ} \mathrm{C}$ é a máxima recomendada para a produção de carvão vegetal, sendo que a partir dessa faixa a celulose e hemiceluloses já foram praticamente degradadas por completo e a degradação da lignina começa a ser mais intensa, o que prejudica o rendimento e as propriedades do carvão vegetal. Acima dessa margem de aquecimento, a produção de carvão vegetal torna-se impactante sob o aspecto ambiental de produção deste bioredutor, devido às elevadas emissões de gases de efeito estufa, como o metano e hidrogênio $\left(\mathrm{CH}_{4} \mathrm{e}\right.$ $\mathrm{H}_{2}$ ). Portanto, a faixa de temperatura elencada acima determina emissões de gases de efeito estufa a níveis razoáveis, dado que o processo de carbonização da madeira não exime por completo estes lançamentos para o meio ambiente.

De acordo com Yang et al. (2007), a ausência de um pico de degradação referente à lignina é devida à sua resistência térmica, uma vez que ela é formada por anéis aromáticos e lhes garantem uma ampla decomposição até a temperatura de $900{ }^{\circ} \mathrm{C}$. Além disso, quando se observa a degradação da lignina, ocorre a liberação de gás metano, dado que há elevado teor de grupos metoxílicos $\left(\mathrm{O}-\mathrm{CH}_{3}\right)$ ligados a seus anéis.

\section{Efeito da temperatura final de carbonização na}

\section{emissão dos principais gases de efeito estufa}

Observa-se que a emissão de $\mathrm{CO}_{2}$ apresentou elevação ao aumento da temperatura final. A liberação de $\mathrm{CO}$ não sofreu influência da temperatura final, com média de emissão de $52,46 \mathrm{Kg}$ de gás / tonelada de madeira seca $(15,34)$. O fato de não se observar o aumento das emissões do gás $\mathrm{CO}$ ao longo dos incrementos em temperatura provavelmente se deve a interferência de outros gases durante a leitura do aparelho. 
Tabela 2. Composição em massa dos gases não condensáveis da carbonização da madeira de Eucalyptus sp. para três temperaturas finais.

Table 2. Mass composition of non-condensable gases from the carbonization of Eucalyptus sp. wood for three final temperatures.

\begin{tabular}{ccccc}
\hline \multirow{2}{*}{ Temperaturas $\left({ }^{\circ} \mathbf{C}\right)$} & \multicolumn{4}{c}{ Fator de emissão $\mathbf{K g}$ de gás / tonelada de madeira seca) } \\
\cline { 2 - 5 } & $\mathbf{C O}_{2}$ & $\mathbf{C O}$ & $\mathbf{C H}_{\mathbf{4}}$ & $\mathbf{H}_{\mathbf{2}}$ \\
\hline \multirow{2}{*}{$\mathbf{4 0 0}$} & $97,14 \mathrm{~b}$ & $43,20 \mathrm{a}$ & $4,50 \mathrm{~b}$ & $0,36 \mathrm{~b}$ \\
& $(25,38)$ & $(21,07)$ & $(13,01)$ & $(14,11)$ \\
\multirow{2}{*}{$\mathbf{5 5 0}$} & $137,15 \mathrm{ab}$ & $56,39 \mathrm{a}$ & $10,50 \mathrm{a}$ & $0,70 \mathrm{a}$ \\
& $(5,51)$ & $(6,19)$ & $(11,27)$ & $(9,61)$ \\
\multirow{2}{*}{$\mathbf{0 0 0}$} & $153,38 \mathrm{a}$ & $57,80 \mathrm{a}$ & $11,35 \mathrm{a}$ & $0,84 \mathrm{a}$ \\
& $(15,95)$ & $(7,34)$ & $(21,94)$ & $(8,75)$ \\
\hline
\end{tabular}

Médias, na coluna, seguidas da mesma letra não diferem entre si, a 5\% de probabilidade, pelo teste Tukey. Valores subscritos entre parêntesis representam o coeficiente de variação da média, em \%

Os gases $\mathrm{CH}_{4}$ e $\mathrm{H}_{2}$ tiveram características semelhantes em termos de emissão, dado que entre as temperaturas de $400 \mathrm{e}$ $550{ }^{\circ} \mathrm{C}$ houve aumento significativo em suas emissões e estabilização em $700{ }^{\circ} \mathrm{C}$. Em vista desses resultados, pode-se considerar que, o comportamento geral de aumento da emissão de praticamente todos os gases ocorre em função do aumento da temperatura de carbonização (CANAL et al., 2016; CARNEIRO et al. 2013; PEREIRA et al., 2013; SHEN et al., 2010; YANG et al., 2007). Desse modo, pode-se considerar que aumentos na temperatura de carbonização da madeira promovem a intensificação do potencial poluidor deste processo.

Outro ponto que se deve considerar é o impacto da temperatura final sobre os potenciais de aquecimento global dos gases de efeito estufa mensurados nesse processo. De acordo com Forster et al. (2007), os potenciais de aquecimento global (PAG) dos gases $\mathrm{CH}_{4}, \mathrm{CO}$ e $\mathrm{H}_{2}$ são, respectivamente, 25, 1,9 e 5,8 vezes maiores que o dióxido de carbono, considerado como gás de efeito estufa referencial $\mathrm{CO}_{2 e}$. Em vista disso, quando se promove o aumento da temperatura de 400 para $550{ }^{\circ} \mathrm{C}$, a emissão de $\mathrm{CO}_{2 e}$ em massa aumenta de 311,40 para 503,37 Kg gás / tonelada de madeira seca. Portanto, os resultados apresentados neste trabalho podem auxiliar para uma produção de carvão vegetal menos impactante sob o ponto de vista das emissões de gases de efeito estufa para o meio ambiente.

\section{Efeito da temperatura final de carbonização}

\section{nos rendimentos dos produtos da pirólise}

O aumento da temperatura final até $700^{\circ} \mathrm{C}$ proporcionou a diminuição do RGCV e aumentos do RGGC e RGNC (Figura 5). Em consonância aos resultados obtidos nesse estudo, vários autores mencionam que existe uma elevação progressiva do rendimento em gases em função da redução do rendimento em carvão (BECIDAN et al., 2007; PINHEIRO; SÈYE, 1998). Para Bianchi et al. (2010) e Pereira et al. (2013), durante a pirólise da madeira os incrementos em temperatura proporcionam uma complexa decomposição térmica dos constituintes da madeira e, consequentemente, variada eliminação de subprodutos na forma líquida ou gasosa. Porém, deve-se considerar que variações nos teores dos elementos constitutivos da madeira também proporcionarão variados rendimentos em gases condensáveis, gases não condensáveis e carvão vegetal. De acordo com Yang et al. (2007), a celulose, hemiceluloses e lignina são provavelmente constituídos de alquenos, ésteres, cetonas, álcoois e aromáticos, com diferentes grupos funcionais, como por exemplo, $\mathrm{OH}, \mathrm{C}=\mathrm{O}, \mathrm{C}-\mathrm{O}-\mathrm{C}$ e C-O- $(\mathrm{H})$, 
o que garante diversas faixas térmicas de transformação da madeira in natura em produtos gasosos e carvão vegetal.

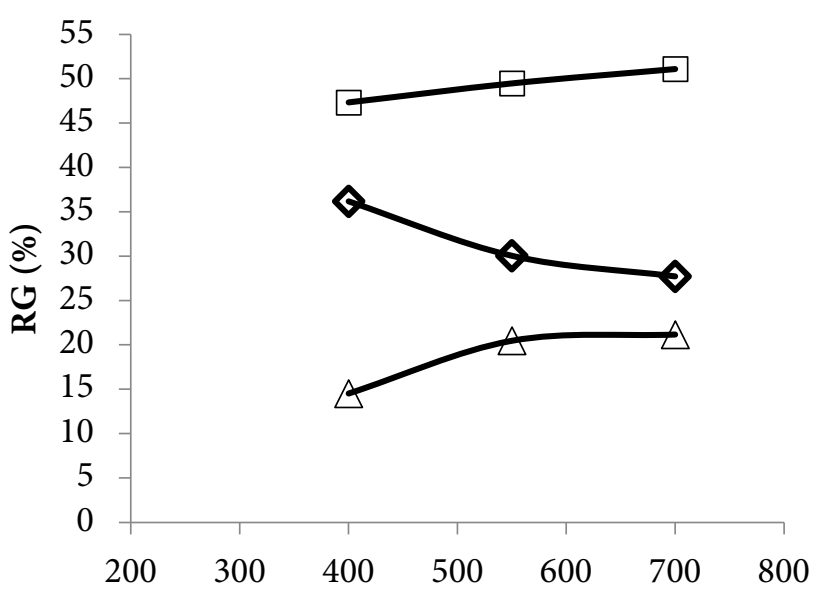

Temperatura final de carbonização $\left({ }^{\circ} \mathrm{C}\right)$

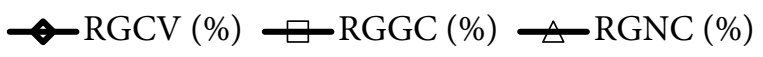

Figura 5. Rendimentos gravimétricos (RG) dos produtos da carbonização da madeira de Eucalyptus sp.

Figure 5. Gravimetric yields (GY) of products from the carbonization of Eucalyptus sp. wood

Em que: RGCV: Rendimento gravimétrico em carvão vegetal, RGGC: Rendimento gravimétrico em gases condensáveis; RGNC: Rendimento gravimétrico em gases não condensáveis.

Durante essa ocorrência, Shen et al. (2010) considera que após a primeira fase de secagem, uma perda abrupta de massa da madeira na faixa de temperatura entre 225 e $430{ }^{\circ} \mathrm{C}$, sendo originada pela quebra da celulose e hemiceluloses. Portanto, para Santos et al. (2012), observa-se que em temperaturas abaixo de $450{ }^{\circ} \mathrm{C}$ ocorre a termodegradação das hemiceluloses e celulose, que são principalmente responsáveis pela liberação de gases condensáveis. Canal et al. (2016) observaram ainda que para essa temperatura há o total exaurimento das hemiceluloses e celulose no corpo da biomassa enfornada.

Portanto, o fator temperatura não proporcionou expressiva desagregação da lignina para a formação de gases condensáveis e não condensáveis, sendo este fato elencado somente para a quebra térmica da celulose e hemiceluloses.

\section{Densidade do carvão vegetal}

Os resultados da densidade aparente do carvão em função das temperaturas de 400, 550 e $700{ }^{\circ} \mathrm{C}$ não diferiram significativamente a $5 \%$ (p-valor $=0,056879)$, observando-se uma média de $0,29 \mathrm{~g} \cdot \mathrm{cm}^{-3}$ (com 10,94\% de variação em torno da média) para todos os tratamentos. Esse valor obtido caracteriza o carvão vegetal como inferior para os padrões de uso siderúrgico, uma vez que, de acordo com Carneiro et al. (2013), a densidade aparente do carvão vegetal deve apresentar valores superiores a $0,4 \mathrm{~g} \cdot \mathrm{cm}^{-3}$.

A densidade do carvão inferior para a utilização siderúrgica é provavelmente reflexo da baixa densidade básica da madeira. Dessa forma, neste experimento a quantidade de matéria carbonizada por unidade de volume foi menor em comparação com madeiras de Eucalipto geralmente utilizadas na carbonização, como por exemplo clones de Eucalyptus urophyla, citado por Protásio et al. (2015).

Em contrapartida, em termos de emissões gasosas, devese pontuar que a utilização de madeiras de alta densidade na carbonização significa em maiores concentrações de carbono, oxigênio e hidrogênio exauridos na forma de gases condensáveis e não condensáveis.

\section{Conclusões}

1) A geração dos gases $\mathrm{CO}_{2}$ e $\mathrm{CO}$ tem tendência de estabilização com o aumento da temperatura, e a geração dos gases $\mathrm{H}_{2}$ e $\mathrm{CH}_{4}$ apresenta crescimento progressivo com o aumento da temperatura;

2) Aumentos sucessivos nas emissões de gases não condensáveis ocorrem de acordo com aumentos em temperatura;

3) Aumentos na temperatura promovem aumentos nos rendimentos gravimétricos em gases condensáveis e gases 
não condensáveis e diminuição no rendimento gravimétrico em carvão vegetal;

4) Incrementos em temperatura não influenciaram na obtenção de carvão vegetal com diferentes densidades.

\section{Agradecimentos}

Os autores agradecem à Fundação de Amparo à Pesquisa do Estado de Minas Gerais - FAPEMIG, ao Conselho Nacional de Pesquisa e Desenvolvimento - $\mathrm{CNPq}$ e à Secretaria de Estado de Ciência, Tecnologia e Ensino Superior - SECTES pelo apoio financeiro; e à Unidade Descentralizada da Empresa Brasileira de Pesquisa Agropecuária - Embrapa Florestas pelo apoio dado para realização desse trabalho.

\section{Referências}

BECIDAN, M.; SKREIBERG, O.; HUSTAD, J. Products distribution and gas release in pyrolysis of thermally thick biomass residues samples. Journal of Analytical and Applied Pyrolysis, Amsterdam, v. 78, p. 207-213, 2007.

BIANCHI, O.; CASTEL, C. D.; OLIVEIRA, R. V. B. Avaliação da degradação não-isotérmica da madeira através de termogravimetria - TGA. Polímeros, Porto Alegre, v. 20, n. especial, p. 395-400, 2010.

CANAL, W. D.; CARVALHO, A. M. M. L.; OLIVEIRA, A. C.; MAGALHÃES, M. A.; CÂNDIDO, W. L.; FIALHO, L. F. Comportamento térmico e emissão de gases não condensáveis no processo de carbonização da madeira. Pesquisa florestal brasileira, Colombo, v. 36, n. 87, p. 261267, 2016.

CARNEIRO, A. C. O.; VITAL, B. R.; OLIVEIRA, A. C.; PEREIRA, B. L. C. Pirólise lenta da madeira para produção de carvão vegetal. In: SANTOS, F.; COLLODETTE, J.; QUEIROZ, J. H. Bioenergia \& Biorrefinaria - Cana-deAçúcar \& Espécies Florestais. 1. ed. Viçosa: Editora UFV, 2013. p. 429-458.

COUTO, A. M.; TRUGILHO, P. F.; NAPOLI, A.; LIMA, J. T.; SILVA, J. R. M.; PROTÁSIO, T. P. Qualidade do carvão vegetal de Eucalyptus e Corymbia produzido em diferentes temperaturas finais de carbonização. Scientia Forestalis. Piracicaba, v. 43, n. 108, p. 817-831, 2015.
DUBOC, E.; COSTA, C. J.; VELOSO, R. F.; OLIVEIRA, L. dos S.; PALUDO, A. Panorama atual da produção de carvão vegetal no Brasil e no Cerrado. Planaltina, DF: Embrapa Cerrados, 2007. 37 p., ISSN 1517-5111. (Documentos, 197).

FORSTER, P. V.; RAMASWAMY, P.; ARTAXO, T.; BERNTSEN, R.; BETTS, D.W.; FAHEY, J.; HAYWOOD, J.; LEAN, D. C.; LOWE, G.; MYHRE, J.; NGANGA, R.; PRINN, G.; RAGA, M.; SCHULZ, R.; VAN DORLAND, R.; BODEKER, G.; BOUCHER, O.; COLLINS, W. D.; CONWAY, T. J.; DUGLOKENCKY, E.; ELKINS, J. W.; ETHERIDGE, D.; FOUKAL, P.; FRASER, P.; GELLER, M.; JOOS, F.; KEELING, C. D.; KEELING, R.; KINNE, S.; LASSEY, K.; LOHMANN, U.; MANNING, A. C.; MONTZKA, S.; ORAM, D.; O'SHAUGHNESSY, K.; PIPER, S.; PLATTNER, G. K.; PONATER, M.; RAMANKUTTY, N.; REID, G.; RIND, D.; ROSENLOF, K.; SAUSEN, R.; SCHWARZKOPF, D.; SOLANKI, S. K.; STENCHIKOV, G.; STUBER, N.; TAKEMURA, T.; TEXTOR, C.; WANG, R.; WEISS, R.; WHORF, T. Changes in atmospheric constituents and in radiative forcing. In: SOLOMON, S. D.; QIN, M.; MANNING, Z.; CHEN, M.; MARQUIS, K. B.; AVERYT, M.; TIGNOR; MILLER, H. L. Climate change 2007: The physical science basis. Contribution of working group i to the fourth assessment report of the intergovernmental panel on climate change. United Kingdom and New York: Cambridge University Press, 2007, v. 1, p. 129-234.

GONZÁLEZ, J. F.; ENCINAR, J. M.; CANITO, J. L.; SABIO, E.; CHACÓN, M. Pyrolysis of cherry stes: energy uses of the different fractions and kinetic study. Journal of Analytical and Applied Pyrolysis, Amsterdam, v. 67, p. 165-190, 2003.

KLOSE, W.; DAMM, S.; WIEST, W. Pyrolysis and activation of different woods: thermal analysis (TG/EGA) and formal kinetics. In: Proceedings of the fourth international symposium of catalytic and thermochemical conversions of natural organic polymers, 1., 2000, Krasnoyarsk. Anais... Krasnoyarsk, 2000.

OLIVEIRA, A. C.; CARNEIRO, A. C. O.; PEREIRA, B. L. C.; VITAL, B. R.; CARVALHO, A. M. M. L.; TRUGILHO, P. F.; DAMÁSIO, R. A. P. Otimização da produção do carvão vegetal por meio do controle de temperaturas de carbonização. Revista Árvore, Viçosa, v. 37, n. 3, p. 557-566, 2013.

PEREIRA, B. L. C.; CARNEIRO, A. C. O.; CARVALHO, A. M. M. L.; TRUGILHO, P. F.; MELO, I. C. N. A.; OLIVEIRA, A. C. Estudo da degradação térmica da madeira de Eucalyptus 
através de termogravimetria e calorimetria. Revista Árvore, Viçosa, v. 37, n. 3, p. 567-576, 2013.

PINHEIRO, PC da C.; SĖYE, O. Influência da temperatura de carbonização nas propriedades do carvão vegetal de Eucalyptus. In: Congresso anual da ABM. 1998. p. 2032.

PROTÁSIO, T. P.; COUTO, A. M.; REIS, A. A.; TRUGILHO, P. F.; GODINHO, T. P. Potencial siderúrgico e energético do carvão vegetal de clones de Eucalyptus spp. aos 42 meses de idade. Pesquisa florestal brasileira, Colombo, v. 33, n. 74, 2013.

PROTÁSIO, T. P.; COUTO, A. M.; TRUGILHO, P. F.; JUNIOR, J. B. G.; JUNIOR, P. H. L.; SILVA, M. O. Avaliação tecnológica do carvão vegetal da madeira de clones jovens de Eucalyptus grandis e Eucalyptus urophylla. Scientia Forestalis. Piracicaba, v. 43, n. 108, p. 801-816, 2015.

RAAD, T. J.; PINHEIRO, P. C. C.; YOSHIDA, M. I. Equação geral de mecanismos cinéticos da carbonização do Eucalyptus sp. Revista Cerne, Lavras, v. 12, n. 2, p. 93-106, 2006.

SANTOS, R. C.; CARNEIRO, A. C. O.; TRUGILHO, P. F.; MENDES, L. M.; CARVALHO, A. M. M. L. Análise termogravimétrica em clones de eucalipto como subsídio para a produção de carvão vegetal. Revista Cerne, Lavras, v. 18, n. 1, p. 143-151, 2012.

SHEN, R.; GU, S.; BRIDGWATER, A. V. The thermal performance of the polysaccharides extracted from hardwood: cellulose and hemicelluloses. Carbohydrate Polymers, v. 82, p. 39-45, 2010.

VIEIRA, R. S.; LIMA, J. T.; MONTEIRO, T. C.; SELVATTI, T. S.; BARAÚNA, E. E. P.; NAPOLI, A. Influência da temperatura no rendimento dos produtos da carbonização de Eucalyptus microcorys. Revista Cerne, Lavras, v. 19, p. 59-64, n. 1, 2013.

VITAL, B. R. Métodos de determinação de densidade da madeira. Viçosa: SIF, 1984. 21 p. (Boletim técnico, 1).

YANG, H.; YAN, R.; CHEN, H.; LEE, D. H.; ZHENG, C. Characteristics of hemicellulose, cellulose and lignin pyrolysis. Fuel, v. 86, p. 1781-1788, 2007. 